Voix et Images

voixetimages

\title{
Entretien avec Michael Delisle
}

Daniel Laforest et Michel Nareau

Volume 38, numéro 3 (114), printemps-été 2013

Michael Delisle

URI : https://id.erudit.org/iderudit/1018308ar

DOI : https://doi.org/10.7202/1018308ar

Aller au sommaire du numéro

\section{Éditeur(s)}

Université du Québec à Montréal

\section{ISSN}

0318-9201 (imprimé)

1705-933X (numérique)

Découvrir la revue

Citer ce document

Laforest, D. \& Nareau, M. (2013). Entretien avec Michael Delisle. Voix et Images, 38(3), 13-23. https://doi.org/10.7202/1018308ar d'utilisation que vous pouvez consulter en ligne.

https://apropos.erudit.org/fr/usagers/politique-dutilisation/ 


\title{
ENTRETIEN AVEC MICHAEL DELISLE
}

\author{
$+++$ \\ DANIEL LAFOREST \\ Université de l'Alberta \\ MICHEL NAREAU \\ Collège militaire royal du Canada
}

voIX ET IMAGES Comment en êtes-vous venu à l'écriture? Il semble que la revue Lèvres urbaines, que vous avez fondée avec Claude Beausoleil en 1983, ait joué un rôle dans ce début littéraire. Est-ce le cas? Aviez-vous déjà entrepris votre travail de création? MICHAEL DELISLE Lèvres urbaines est vraiment, dans mon histoire bibliographique, un épiphénomène, ce n'est pas important du tout. Je viens d'une génération où il n'y avait pas de modèles littéraires comme aujourd'hui. À la fin des années 1970, il n'y avait pas d'écrivains vedettes au Québec, je n'avais pas d'image claire de cette réalité. C'était même difficile de trouver, à Longueuil, non seulement un roman québécois, mais un roman tout court [rires]. Je pars de loin. Écrire, je faisais ça pour moi, sans penser qu'il en adviendrait quelque chose. C'est au cégep que m'est venu le premier contact avec la littérature et la poésie québécoises. Claude Beausoleil était mon professeur de poésie. Avec lui, j'ai vu qu'un écrivain n'était pas nécessairement une figure mythique sortie du XIXe siècle. Un écrivain, ça existait, et publier, c'était faisable; c'est lui qui m'a montré qu'on pouvait publier. Le problème est que j'écrivais en secret des textes très éloignés du postformalisme ludique qui faisait rage dans son cercle. Ce que je faisais à seize ans était modelé sur des fables de La Fontaine. Je ne viens pas d'un environnement où la littérature avait sa place. Il n'y en avait pas chez moi. Ma mère était acculturée par ses origines anglophones et mon père était rarement présent : leur absence respective avait créé un désert culturel assez opaque. C'est au cégep que j'ai commencé à voir les avenues possibles pour mon désir d'écriture. Puis, grâce à Beausoleil, j'ai rencontré des écrivains, assisté à des lancements. J'étais assez habile et ambitieux pour savoir comment faire, mais mon propos ne trouvait pas sa place dans - là, on doit se replacer à la fin des années 1970 - un discours marxiste, formaliste ou psychanalytique. C'est peut-être cette circonspection qui explique autant l'économie extrême de mes premiers textes (colonnes de mots-phrases) que leur aspect « collage». C'est l'époque des recueils comme L'extase neutre, où le genre changeait à chaque page, de mes collaborations à Hobo-Québec. Je voyais le livre comme une boîte où j'entassais des morceaux. Graduellement, ça s'est épuré pour aller vers un genre dirigé, mais mes premières années ont été un peu joyeusement n'importe quoi. On ne doit pas oublier que j'ai commencé à publier dans un mouvement de modernité où le texte (comme hybride générique) et le mélange des genres étaient 
fréquents, donc, de mon point de vue d'aspirant, autorisé. Cette ouverture a favorisé mon écriture. Ça m'a permis de ne pas avoir à choisir. J'ai tout pris. J'ai été proche de Beausoleil pendant une dizaine d'années. La vie que je menais dans sa proximité faisait en sorte que je percevais la publication comme étant de l'ordre du privé. La revue Lèvres urbaines était presque une activité domestique. La littérature était centrale dans mon quotidien; on colligeait des recueils comme d'autres écoutent la télé après le souper. On improvisait des lectures. C'était une vie littéraire très incarnée, mais dans un cercle assez restreint pour que, de mon point de vue, ça ait - je déteste le mot, mais il s'impose - une connotation familiale. Mes premières plaquettes ont vu le jour grâce à des éditeurs qui venaient souper à la maison et qui me demandaient: «As-tu quelque chose?» Je n'ai donc pas eu un premier livre qui m'a projeté dans le social. Je ne me souviens pas d'un événement premier. Ça a été graduel. Ça se passait à la maison et Lèvres urbaines faisait partie de cette vie. Beausoleil disait: «Il y en a qui s'achètent des voitures; nous, on fait une revue de poésie.» Je tapais les textes moi-même à la machine, puis on allait porter ça en autobus chez un imprimeur sur le boulevard Saint-Michel, ensuite on allait les porter dans les librairies. Ça m'a pris du temps avant de prendre conscience du sens réel de la publication, c'est-à-dire devenir public à travers ce que j'écris. C'est une expérience que je n'ai réellement sentie qu'à mon septième ou huitième titre.

voıX ET IMAGES L'expérience d'écrire publiquement est-elle venue en partie avec le mémoire de maîtrise en création littéraire que vous avez fait à l'Université du Québec à Montréal ? Ou la prise de conscience s'est-elle plutôt faite à l'extérieur de ce cadre-là ? MICHAEL DELISLE Mon mémoire de maîtrise en création n'est pas très bon. Je me suis retrouvé à l'UQAM dans un programme où on devait faire une partie fiction puis une partie théorique, et les relier s'est révélé une tâche pénible pour une raison particulière : j'ai élaboré un projet de création (un passage de la prose à la poésie à l'intérieur d'un même texte) pour me rendre compte que je l'avais déjà réalisé un an plus tôt (avec Drame privé). À ce moment-là, tout a bloqué et après maintes prolongations, j'ai achevé la chose pour m'en débarrasser. Feu Noël Audet, mon directeur de mémoire, trouvait que je n'avais pas besoin d'aide, alors je n'en ai pas eu. Cela dit, nos rapports étaient conviviaux. Les études en création littéraire ne m’ont pas servi à devenir écrivain; j'ai commencé le programme alors que j'avais déjà plusieurs titres publiés et ce statut m'ostracisait manifestement. Aujourd'hui, je me rends compte que ces études ont leur utilité. Je m'y suis rapaillé un coffre à outils pour régler certains problèmes d'écriture. Quand je m'embourbe dans un chapitre, je peux faire un découpage déictique, un schéma narratif, etc. Mais faire une maîtrise en création littéraire n'a pas fait de moi un écrivain, non. Cela m’a donné des outils pour négocier des situations éditoriales, comme lorsqu' on me demande de changer la fin d'un texte et que je refuse ; je suis capable de défendre l'état du texte, sa nécessité, et je suis capable de le faire avec autorité. Mais c'est à peu près tout.

VoIX ET IMAGES Mais cette maîtrise en création littéraire n'a-t-elle pas coïncidé avec le moment où s'est faite la sortie de l'édition un peu familiale pour aller vers la production plus instituée?

michael DelisLe Non. Ça a commencé avec Fontainebleau. À partir de ce titre, mes publications deviennent des livres, non pas ce qu' on appelait «du laboratoire», 
mais des œuvres avec une identité nette. Avant, c'étaient des plaquettes, surtout. Fontainebleau - c'est étonnant que cet objet ait reçu un prix de poésie - est aussi le premier de mes livres qui, malgré son éclatement apparent, repose sur une proposition. C'est un livre né d'une chose à dire, d'une chose à montrer. Les recueils précédents étaient plus ludiques ou bien justement fragmentés par la crainte de la proposition, comme une phrase qu'on n'ose pas finir.

VoIX ET IMAGES Vous avez débuté avec l'artisanal, parce que vous avez tout essayé de l'expérimentation. Mais on trouve encore dans Fontainebleau, qui est un livre fort et plus substantiel, un travail très conceptuel, dans lequel vous mélangez la prose avec des expérimentations de voix pas nécessairement prosaïques. Et au centre de tout cela, il y a la photographie. Celle-ci revient aussi, périodiquement, dans vos préoccupations. Quelle importance l'image a-t-elle eue pour vous à ce moment-là, et durant votre carrière d'écrivain dans son ensemble?

Michael Delisle Dans Fontainebleau, les photos s'appellent des «certitudes». C'est un peu ça. J'avais en tête ce que Ludwig Wittgenstein en dit: la certitude est de la nature d'un ton dans la voix, elle n'est aucunement gage de vérité. Ce sont des certitudes parce que les photos encadrent, limitent. Elles finissent l'image. Les photos ressemblent à l'écriture dans la mesure où, comme les Anciens le disaient au sujet de l'hébreu, elles sont une sorte de matériau mort qui s'anime avec le souffle du lecteur. La photo n'est rien, c'est sa lecture qui en fait un être de langage. On me rapporte souvent que Fontainebleau est efficace dans les cours de création littéraire parce qu'il donne la permission de tout prendre, de tout faire, et d'utiliser l'enfance comme réservoir de fiction. L'enfance est une source féconde ; c'est la banque des "premières fois ", où les événements sont souvent cimentés par le non-dit. Il y a donc à redire. Les photos, surtout anciennes, sont utiles pour débloquer la mémoire et pour traverser l'angoisse de la page blanche. On décrit la photo et le réchauffement se fait, le texte commence, est déjà commencé... En fait, Fontainebleau donne le goût d'écrire. C'est ce qu'on m'a dit du moins. Et ça ne m’étonne pas.

VoIX ET IMAGES Est-ce qu'il y a une photo qui serait centrale dans le projet de Fontainebleau? Parce que sur la couverture de l'édition des Herbes rouges, il y a la photo de cette femme assise dans une posture étrange, projetant ses bras vers l'arrière, sur un perron de banlieue, avec un petit garçon entre ses jambes.

MICHAEL DELISLE Sur cette photo, il y a ma mère et mon frère. La photo montre l'enfant entre les jambes de la femme, qui est manifestement coupée de la conscience de sa présence. Ce n'est pas la Vierge à l'enfant, c'est un geste de séduction qui exige au contraire qu'on oublie l'enfant un instant. Yolande Villemaire, devant l'étalage des photos sur ma table de travail, m'avait dit d'utiliser celle-là pour la couverture. C'est vrai qu'il y a quelque chose d'un peu star sur cette photo, et Yolande y a sûrement été sensible. J'ai suivi son intuition et, avec le travail, la photo est devenue l'emblème du livre. C'est donc après coup que la prégnance de cette photo m'est apparue.

voix ET IMAGES Cela rappelle La chambre claire de Roland Barthes, où il y a la mère qu'on finit par retrouver dans la photographie. Nous nous demandions si la figure maternelle était un peu venue s'imposer dans ce livre.

MiChAEL DELISLE Je me demande si les portraits de femmes sont plus nombreux que les portraits d'hommes parce que photographier est un geste viril: on vise, on case. 
Ça repose sur une pulsion de chasse. J'ai toujours trouvé naturel que les photos de mon père n'apparaissent pas dans la boîte familiale. Par ailleurs, la figure maternelle est peut-être surprésente dans mes textes parce qu'il y a beaucoup à redire à son propos. C'est une figure féconde. Ma mère dit toujours: «Si je n'étais pas là, tu n'aurais rien à dire» [rires]. C'est un petit peu vrai. Le souvenir de mon père, bizarrement, a toujours inspiré une voix du côté de la poésie, ou du discours psychopathologique, comme dans Le désarroi du matelot, qui est très proche de son histoire. Je pense que son absence fondamentale me mène naturellement du côté de la poésie. Avec ma mère, au contraire, je suis davantage du côté du storytelling, peut-être à cause de son côté anglais. Ma grand-mère maternelle était une bonne conteuse. Bref, si je parle de ma mère, c'est un roman qui s'impose. Avec mon père, c'est de la poésie, ou un discours narratif poétique, c'est-à-dire schizophrénique. C'est peut-être le thème, d'une part, et la forme, d'autre part, d'une même sociopathie.

voIX ET IMAGES C'est intéressant. Cette distinction entre les genres renverse le cliché de la poésie comme langue orale, comme langue maternelle, et toutes ces associations de la poésie à la dimension maternelle.

MICHAEL DELISLE Je ne suis pas à une inversion près [rires] !

VoIX ET IMAGES Une autre chose frappe avec votre dernier recueil publié, qui est magnifique : Prière à blanc. On y voit une dimension spirituelle, mais pas nécessairement religieuse. Il y a une certaine aspiration à l'ascèse, une tension vers la voix intérieure. Dans vos préoccupations, dans votre œuvre, cela vient faire un contraste avec cet autre univers qui est celui de la brutalité de la vie matérielle, des objets. On pense alors davantage à vos romans, comme Dée.

MICHAEL DELISLE L'abjection.

VoIX ET IMAGES L'abjection, oui... On pourrait employer le mot "prosaïque » pour qualifier ces textes?

MICHAEL DeLISLE Mais c'est encore la mère. La poésie, pour moi, est vraiment du côté du père, et je pense que son absence physique dans ma vie est pour beaucoup dans la propreté de mes poèmes. C'est un homme religieux. Il lit la Bible du soir au matin; il fréquente un groupe évangélique. C'est un mystique très occupé. C'est mon héritage aussi. Comme lecteur, je n'aime pas la critique biographique, mais pour l'écrivain que je suis, ces liens sont d'autant plus évidents que je donne, de plus en plus, dans le roman familial. Ma pratique tente justement de thématiser cet héritage. J'ai commencé à écrire en faisant une sorte de mélange de genres, puis j'ai fini par distiller les deux. Je me rends compte que je fais une poésie très poétique, et des romans qui sont, comme dit ma traductrice, Gail Scott, «so realist...» [rires].

VoIX ET IMAGES Dans vos romans, on retrouve assurément ce côté-là, parfois presque naturaliste.

Michael DeLISLE Il y a une polarité «ciel et terre», oui. Dans la prière, l'énonciation est particulière. Le précatif m'intéresse beaucoup, comme tous les performatifs liés à la prière : souhaiter, invoquer, espérer. Ce sont des actes de langage qui sont de nature poétique. Ça m'inspire énormément. Le texte des prières me donne souvent une amorce pour écrire de la poésie, même si ma poésie n'est pas religieuse. Comme on écoute certaines musiques pour "se partir», certaines oraisons me donnent le la, me 
montrent que le genre de pureté qu'offre ce discours est faisable. Mais vous avez raison, ce n'est pas religieux, c'est linguistique.

VoIX ET IMAGES En opposition à cela, vous semblez avoir aussi besoin de l'espèce de réalisme brutal dont on a parlé, qui court partout dans votre œuvre, même parfois dans la poésie.

MiChAel Delisle Oui, certainement. Dans Prière à blanc, je voulais prendre plaisir à voir tout ce qu'il était possible de faire avec un poème: un art poétique, du rythme pur, un poème de «navire» comme tout le monde en faisait au XIXe siècle, un poème en prose... Dans ce sens, le «réalisme brutal » s'est invité parce que c'est un possible de la poésie. Mais je n'imagine pas, même s'il peut m'arriver de le souhaiter comme apaisement, qu'un jour ces deux tendances se réconcilient. J'aimerais faire des romans poétiques foisonnants, mais ça ne marche pas. J'aboutis à une prose carrée et réaliste, ou d'un autre côté, à une poésie hermétique parce que poétique.

VoIX ET IMAGES Est-ce que cela demande beaucoup de travail? C'est une drôle de question; nous ne voulons pas sous-entendre que vous ne travaillez pas, mais est-ce difficile? Est-ce qu'une épuration très longue est nécessaire pour en arriver, par exemple, à votre prose? Parce qu'elle donne l'impression de couler de source... C'est une écriture extrêmement maîtrisée.

MichaEL DELISLE Ça représente des années, mais j'ai le luxe de pouvoir me permettre ce temps. Si je vivais uniquement de mon écriture, je serais étonnamment maigre! Dans mon dernier roman, certaines pages ont été écrites il y a trente ans, ou vingt ans. C'est un peu comme un artiste qui ébauche une main. Il la dessine sans savoir dans quel tableau elle aboutira, puis vingt ans plus tard, il ressort le croquis et le place dans un plus grand tableau. Je suis un écrivain lent. Il est rare que je montre des textes qui n’ont pas décanté le temps qu'il faut.

voix ET IMAGES Cette lenteur est-elle liée à des pauses fréquentes dans la rédaction ou à un travail imposant de réécriture?

MICHAEL DELISLE C'est une précaution que je prends parce que mon propos est souvent personnel et il m'importe que le résultat soit littéraire. Je ne veux pas que le livre soit l'expression d'un sentiment. Je n'ai jamais eu l'expression comme objectif. Je veux que la finalité soit littéraire, et l'épuration est faite à cette fin. C'est un travail de distillation. Je dis que je fais actuellement mon «autobiographie», mais il est certain que le résultat n'aura rien à voir avec le genre autobiographique. Je n'imagine pas écrire un livre de témoignage, mais il est vrai que mon propos s'y apparente, et cette proximité m'oblige à ces précautions. Je retiens le livre le temps qu'il faut pour m'assurer qu'il devienne objet.

VoIX ET IMAGES Il y a un travail d'épuration non seulement dans le style, mais dans le rapport à l'action aussi, surtout dans les proses. Il y a beaucoup d'ellipses, de nondits dans vos textes. Les choses y sont suggérées plutôt que d'être présentées. Il est vrai qu'on vous associe souvent à la représentation crue des choses, celle du quotidien, du banal, mais il y a quand même beaucoup de flottement dans vos descriptions.

Michael DelisLe Ce sont des romans écrits par quelqu'un qui a une expérience de la poésie, qui sait qu'un mot isolé sur une page peut marcher si c'est le bon, et que l'ellipse, le blanc, le silence peuvent être aussi parlants qu'une phrase. J'aime penser que mes livres rendent le lecteur créateur. Je travaille à laisser une place au lecteur en lui 
présentant un narrateur estompé en quelque sorte (par sa culture souvent), avec des phrases un peu plus simples, avec des mots qui s'approchent de l'icône. C'est ça, c'est une façon de laisser toute la place à la lecture.

voix ex IMAGes Dans Chose vocale, vous disiez que c'est le propre de la poésie que de se placer dans ce rapport-là, où on en demande beaucoup au lecteur.

MICHAEL DELISLE Décidément, on revient à l'UQAM! Ce livre date de mes recherches sur le genre littéraire. J'en étais venu à trouver que la différence essentielle entre la poésie et la narration est la notion d'altérité utilisée pour définir des concepts fondamentaux comme l'espace, le temps, le lieu et le sujet. Dans ce système, les lieux du roman se définissent par un autre lieu: ici et ailleurs, tandis que dans le poème pur, le lieu serait souverain, non défini par une notion autre; il n'y a pas d'autre vaisseau dans Le vaisseau d'or. Même chose par rapport au temps romanesque : présent, passé, futur. Dans la poésie, je voyais une sorte de présent perpétuel, comme un point qui avance au fur et à mesure de la lecture, sans chronologie. J'avais finalement trouvé des exemples (de la Bible à Anne Hébert) où je pouvais appliquer ce principe au sujet. Le «je» du poème n'est pas un «je» détouré par une notion d'altérité, comme le «tu», le «il». Et s'il le devient, c'est parce qu'il se déplace vers le roman. J'avais mis en place un système d'aiguillage pour savoir si on est plus dans le roman ou dans le poème, comme pour savoir si on est plus au nord ou au sud. Je crois que c'est à partir de ces épiphanies [rires] que je suis résolument sorti du mélange pour commencer à séparer les genres. Ma poésie est devenue une sorte de langue souveraine. Le «je» de mon poème ne veut rien dire, mais le «je» de mon roman est porteur de caractère. Le «je » du poème, pour moi, c'est une clé musicale, comme chanter en do, ou chanter en fa. VoIX ET IMAGES Déplaçons-nous un peu vers une autre préoccupation, à savoir l'histoire culturelle récente. Indéniablement, une chose frappe dans votre œuvre: il y a une démarche d'autofiction...

MiChAEL DELISLE Oui, le travail que je fais en ce moment est de ce côté. C'est immédiatement sous mon titre de travail. Je veux explorer ce que j'ai à faire dans l'autofiction. En même temps, j'ai tellement de préjugés contre ça. J'y vois une connotation de paresse.

VoIX ET IMAGES Quoiqu'il en soit, il y a toute cette démarche, avec le temps personnel, le temps familial, l'histoire familiale. Et à côté, comme dans Fontainebleau, il y a Ville Jacques-Cartier, qui est devenue Longueuil. Les souvenirs familiaux sont enracinés, mais pas n'importe où. Ils ne le sont pas dans des monuments, des symboles. On remarque plutôt un intérêt pour ce qu'on peut appeler les aspects oubliés de la Révolution tranquille, ceux dont on n'a pas beaucoup parlé. On le voit dans Tiroir no 24 aussi, où sont nommées des marques de nourriture très prosaïques, très populaires. Ça fonctionne, ça crée un univers tout de suite. Le ton de toute une époque est donné, mais pas avec les éléments habituels.

MICHAEL DELISLE Mon travail a pris du temps avant de se nicher dans une sorte de créneau institutionnel, mais mon histoire ressemble à ça: ni anglais ni français; banlieue qui n'est ni la campagne ni la ville; l'orphelinat où je n'étais pas orphelin. Je me suis toujours demandé : est-ce que la langue de mon corps est anglaise ou française? L'histoire de mon nom est longue aussi. Ce n'est pas innocent si je m'intéresse aux questions d'identité. Mon œuvre est un reflet de cette vigilance. Fontainebleau, est-ce 
un poème, est-ce un roman? Ça a d'ailleurs été un gros problème avec la diffusion de ce livre. C'est un miracle que j'aie épuisé mon premier tirage, parce que les librairies ne savaient pas où le ranger sur les rayons. Et c'est à l'image de mon parcours qui a commencé dans les marges, dans la marginalité. Je n'aurais pas pu commencer autrement que dans l'avant-garde, parce que c'était la seule place qui admettait tout, y compris les identités sans assise. J'envie les écrivains comme Louis Hamelin et ceux qui sont capables de brandir des figures d'Amérindiens. Ils ont leur créneau; je suis content pour eux. Mais il a fallu du temps avant que la banlieue soit reconnue comme digne de fiction. Tout le monde connaissait la banlieue, mais personne n'en parlait.

voix ET IMAGEs Personne ne pensait que c'était un sujet littéraire. À vrai dire peu le pensent encore aujourd'hui.

MiCHAEL DeLISLE C'est plate, il n'y a rien.

voix ET IMAGES C'est plate, mais l'écrivain est capable de parler de l'ennui et de la platitude. C'est un défi par contre.

Michael delisLe C'est ça. Le défi, c'est de raconter la banlieue où il ne s'est rien passé, avec des personnages qui n'ont pas les mots, avec une culture faite de gens qui n'ont pas de culture. Je ne me rends pas les choses faciles [rires]!

VoIX ET IMAGES Est-ce qu'il faut nécessairement passer par la perspective d'un enfant pour pouvoir décrire cette banlieue-là? Que ce soit dans Helen avec un secret, dans Dée ou dans Fontainebleau, le point de vue est celui d'un enfant.

MICHAEL DELISLE D'un adolescent souvent; un adolescent attardé. C'est peut-être l'aspect autofictif qui fait ça. C'est l'âge que j'avais quand j'y vivais. Je crois que c'est lié à la mémoire. Je ne sais pas ce qu'une voix adulte peut dire de Ville Jacques-Cartier, ça fait trente ans que je n'y ai pas mis les pieds. Je ne sais pas comment ça se passe aujourd'hui. Je ne sais pas ce qu'un écrivain de la banlieue pourrait dire de la banlieue aujourd'hui.

voIX ET IMAGES Vous parliez de Louis Hamelin. La constellation du lynx, sur la crise d'Octobre, se déroule à Saint-Hubert, avec des bungalows et tout. Il y fait un travail d'archéologie de l'avant-hier, que vous faisiez déjà auparavant. Cette perspective a été très tôt présente dans votre œuvre.

MichAEL DELISLE C'est ma mémoire.

voix ET IMAGES C'est votre histoire. Vous avez choisi d'en parler sans laisser de côté les éléments ennuyants.

Michael delisLe L'ennui est un thème, avec les enfants. L'enfance est longue. Dans Fontainebleau, les enfants n'ont rien à faire. Dans Le sort de Fille, les adolescents n'ont rien à faire, puis il y a un projet, qui ne marche pas, et c'est ça l'histoire. C'est un défi auquel je fais face encore en ce moment à cause de mes mémoires. Raconter sa vie quand on a passé le plus clair de son temps assis devant un clavier à taper, c'est pas mal... un défi [rires]!

voıX ET IMAGES Dans l'Histoire de la littérature québécoise qui a été publiée en 2007, la dernière en date, par Michel Biron, François Dumont et Élisabeth Nardout-Lafarge, votre nom arrive chronologiquement avec les années 1980, vous êtes associé aux jeunes écrivains de l'avant-garde urbaine caractérisés par l'espèce de violence, de bouillonnement urbain qui a beaucoup été lié aux Herbes rouges par exemple. Pourtant, l'urbanité chez vous est complètement différente. Peut-être n'a-t-on pas 
vraiment la grille de lecture pour concevoir et accueillir votre projet, qui est de construire un espace périphérique.

MICHAEL DELISLE Dans un projet totalisant comme celui de l'Histoire de la littérature québécoise, il faut classer, mettre quelqu'un quelque part. Les listes dans lesquelles on me classe m'étonnent toujours. Mais ce n'est pas mon rôle d'aller leur dire où me mettre. J'écris, je ne me classe pas. C'est vrai que j'ai évolué dans un socius, un groupe où l'urbanité était le thème emblématique. Beausoleil imposait cette thématique, dans un esprit antinationaliste.

VoIX ET IMAGES Cette mouvance était ouvertement antinationaliste?

Michael DelisLe Plus précisément, ce mouvement participait d'une sorte d'antiterroirisme mis à jour. Il ne fallait surtout pas que se retrouvent le mot «neige» ou le mot «fleuve» dans nos poèmes. Il y avait une tranche de l'avant-garde qui prenait pour cible ce qui avait atteint le statut d'establishment et, pour les tenants de l'urbanité à tout prix, ça représentait, par exemple, l'Hexagone et ses thèmes. Comme je frayais avec ces gens, et à cause de recoupements bibliographiques (mêmes revues, par exemple), j'y ai été associé. Mais même dans ce groupe, ce que je faisais ne ressemblait à rien. On me le disait tout le temps, surtout les éditeurs: "Ça ne ressemble à rien ce que tu fais!» Je me demande encore si c'était un reproche.

voIX ET IMAGES Pour revenir une dernière fois à la banlieue, il y a aussi l'idée qu'elle est un espace dépourvu de culture. Ou alors il y aurait une culture, mais qu'on est incapable de nommer, ce qui fait qu'elle est vue à travers son absence, comme un vide. On décrit les dépotoirs dans Dée, le terrain de stationnement du centre d'achat dans Helen avec un secret. Néanmoins, on voit ici et là apparaître des parcelles de culture qui émeuvent. Par exemple, le frère voué à ses miniatures dans la nouvelle «Terre en friche». Il semble assez important pour vous qu'il y ait un contrepoint culturel au sein de cet univers décrit comme privé de culture.

Michael DeLISLE Oui. On voit le côté essentiel de la culture. Une des problématiques de la banlieue, telle que je la décris, c'est qu'en plus de l'absence de vie culturelle, il y a éradication de sa contrepartie: la nature. Les romantiques avaient compris l'importance de la nature. Je pense que dans Dée je voulais montrer cela. Il y a de la nature, il y a des trous d'eau, il y a des animaux, ça bouge, ça pousse. Du jour au lendemain, tout est repoussé pour étaler du gazon, dresser des bungalows, et c'est une perte. La banlieue est une construction, mais il y a certainement désolation quand on perd la nature sans gagner de vie culturelle. En fait, on devient prisonnier d'une sorte d'équateur entre deux pôles: la nature qui permet l'accès à Dieu (c'est la prescription des romantiques) et la ville qui, par sa densité, force le rapport à l'autre, à l'humain. Avec la banlieue, il n'y a donc ni Dieu ni "prochain». Il n'y a rien: pas de nature, pas de culture. Alors les "parcelles de culture», comme vous les appelez, deviennent, oui, des étincelles d'espérance. Les artistes qui sont issus de la banlieue, de ce vide, en tout cas ceux de ma génération, s'en sont extirpés avec un projet très fort, avec une soif de culture anormale et une ambition dopée qui demeurent très opposées à leur milieu d'origine.

VoIX ET IMAGES Jacques Ferron habitait Ville Jacques-Cartier.

MiCHAEL DeLISLE Oui, le «fils de notaire». Ferron est un bourgeois qui a abouti là. Ce n'est pas un produit de Ville Jacques-Cartier. C'est un père missionnaire qui a trouvé 
son Afrique sur la Rive-Sud. Il était notre médecin de famille. Je me souviens de son bureau qui était plein de papiers, des montagnes de papier qui l'encadraient. Il levait brièvement les yeux puis tendait sa prescription. Pas d'auscultation, pas d'examen [rires]. En sortant, ma mère me disait: «Il est écrivain.» C'était l'explication. Ma première image de l'écrivain, c'était le désordre et l'excentricité. Une image de somme aussi. Je ne sais pas si c'est ma perspective d'enfant, mais il me semble que les piles montaient haut et je pense que mon souvenir en a rajouté. C'était toute une «étincelle d'espérance» dans l'univers beige de la banlieue. Je le voyais vraiment comme une sorte de Merlin, dans mon regard d'enfant. Mais Ferron n'est pas un produit de Ville Jacques-Cartier.

VoIX ET IMAGES Et Pierre Vallières?

michael delisLe Lui, davantage, je crois. Je l'ai relu avant d'écrire Dée, justement pour l'histoire des maisons qu' on doit reculer pour les aligner sur la rue, que j'avais vue d'abord chez Ferron et dont je me souvenais vaguement. Vallières est certainement plus proche de ce que j'ai connu comme personnages à Ville Jacques-Cartier. Ferron disait toujours que le problème de Ville Jacques-Cartier, c'est qu'il n'y avait pas de notables. C'était vrai. On dirait qu'il n'y avait que des bandes, des mauvais garçons. La pègre était très présente; on appelait ça le Far West de Montréal.

VoIX ET IMAGES Nous voudrions maintenant aborder la question de la langue. On voit un peu partout dans votre œuvre des éléments de dualitê linguistique, entre le français et l'anglais. Mais le rapport entre le français et l'espagnol est aussi évoqué dans Drame privé. Est-ce que la mise en scène des dualités linguistiques est une chose qui devait advenir dans votre œuvre? Est-ce un élément qui vous a semblé important, pour être en définitive si récurrent?

MICHAEL DELISLE J'ai toujours en tête la phrase de Sartre: «On parle dans sa langue maternelle, mais on écrit dans une langue étrangère. » Le français que j'écris est une langue étrangère, étrangère au corps, étrangère au réel, et c'est dans cette posture que je travaille. Il est certain que le temps que je prends pour écrire est nourri d'une inquiétude au sujet de la qualité de cette langue. On ne parle pas comme ça, on ne vit pas comme ça, mais c'est bien qu'il y ait cette étrangeté. Il n'en va pas ainsi des dialogues, qui vont souvent plomber cette perfection, salir le vernis clinique du texte. Je me vois comme utilisant un français correct avec des segments de dialogues qui rappellent la réalité et sa vulgarité essentielle. Mais c'est encore là une dualité. Je n'arrive jamais à une langue de fusion qui amalgame tout. Pour l'instant, c'est vraiment une question d'équilibre. Je demeure toujours conscient que l'univers n'est pas français, il est aussi espagnol et anglais. J'ai été élevé par une mère qui me parlait indifféremment en anglais ou en français, sans hiatus de quelque nature que ce soit. J'ai rapidement intégré la relativité du français.

voIX ET IMAGES Est-ce que votre prénom se prononce «Michaël» ou «Michael» [à l'anglaise]?

Michael DelisLe Jamais Michael, même si c'est mon vrai nom. L'officiel, c'est Michael, mais on m'a toujours appelé Mike. Donc je n'ai jamais entendu Michael en tant que tel. Quand je suis entré à l'école catholique, on m'a appelé Michel. J'étais Michel Délisle (avec un «é» qui me faisait horreur). Quand j'ai commencé à écrire, les gens ont prononcé Mi-ka-el Delisle et j'ai laissé faire. C'est resté. Je pense même que c'est 
Beausoleil qui a commencé ça. Il m’a donné mon nom. Mais si tu dis Mi-ka-el Delisle à mon père, il ne saura pas qui c'est. Pour lui, je suis Mike. Ces pérégrinations sont à l'image des identités bousculées qui sont fréquentes dans mes romans. Ça m'a toujours intéressé, le nom propre, l'onomastique. Michael (Mi-ka-el), c'est ce que j'ai construit au fil des ans pour rapailler mes avatars (Mike, Michel, Michael). C'est une réparation. Comme le sont souvent les noms de plume.

voIX ET IMAGES Peut-être est-ce une question piégée, mais est-ce que vous vous voyez comme un écrivain québécois? Est-ce que vous considérez votre œuvre comme faisant partie de la littérature québécoise? Est-ce que cela a un sens particulier à vos yeux ? MiCHAEL DeLISLE Je suis un écrivain québécois. Cette phrase est arrivée tard dans mon cheminement. C'est le commentaire sur mon œuvre qui a cristallisé cette conscience, parce que, marginalité oblige, je ne la sentais pas au départ. J'ai rapidement intégré que j'étais «celui qu'on ne sait pas où mettre ». Et j'avais le luxe de faire partie d'une génération qui pouvait, pour la première fois peut-être, ne pas porter le joug de la problématique. Si j'avais commencé à écrire en 1963, je crois que la question aurait été incontournable. Mais le commentaire universitaire, les essais sur la littérature qui ont pour objet de placer l'écrivain dans un groupe ont fini par montrer mon travail d'un point de vue nouveau pour moi. La marginalité de mes origines (personnelles et littéraires) a fait en sorte que je n'avais jamais pensé appartenir à un groupe ; j'ai toujours été en marge du groupe. C'est également mon histoire familiale. Mon frère et moi étions des enfants à qui beaucoup n'avaient pas la permission de parler pour toutes sortes de raisons, la principale étant que mon père était une figure connue de la pègre locale [rires]. On s'est habitués à l'isolement. Être écrivain québécois est donc venu avec le regard de l'autre, je dirais même avec le regard universitaire sur mon travail.

VoIX ET IMAGES Mais aujourd'hui, est-ce que vous y tenez, à cette identité d'écrivain québécois? Vous ne semblez pas vouloir être associé à ce que d'autres conçoivent comme une francophonie internationale.

MICHAEL DELISLE J'ai mon histoire et je reste, en dépit de cet isolement, un produit de mon pays. L'identité québécoise d'accord, mais seulement si c'est une réalité vivante, c'est-à-dire en croissance. Cela dit, je ne me vois pas du tout comme archétypal. Je ne pense pas que Dée le soit [rires]. Ce n'est pas un hasard si mon propos commence à rayonner seulement maintenant. Je le remarque au nombre de commentaires universitaires, à l'intérêt de cinéastes, aux réimpressions. Ça fait trente ans que je publie, mais la réception institutionnelle que mon travail connaît actuellement est relativement récente. Je trouve intéressant de voir que des personnages comme Dée commencent à trouver leur place dans un corpus aussi traditionnel, aussi fermé que celui de la littérature québécoise. J'ai récemment travaillé sur la figure des Chinois de Montréal pour un projet de nouvelle. Ils m'intéressent beaucoup. Je trouve inadmissible qu'en littérature québécoise, on en ait si peu parlé. Il y a des Chinois à Montréal depuis le milieu du XIX siècle et pas un, que je sache, n'a fait son chemin dans la fiction romanesque avant 1980. C'est une absence louche. Il n'y a rien d'innocent à ce que maintenant, après le déblocage survenu avec l'écriture migrante dans la littérature québécoise, des personnages culturellement hybrides, comme Dée, soient considérés. J'avais tout ce qu'il faut pour écrire ce roman en 1986, mais ça a plutôt donné 
Fontainebleau. Dée n'aurait pas passé, je pense. À l'époque du Matou, des Filles de Caleb... ça n'aurait pas été reçu [rires]. J'ose espérer que la fortune actuelle du roman n'est pas liée au ramassage de miettes d'une identité nationale qui se sent en péril.

VoIX ET IMAGES Il y a beaucoup d'écrivains, peut-être de plus en plus, qui sont manifestement passés par la machine universitaire, qui ont lu Derrida, et cela paraît dans leurs livres. Vous possédez également ce bagage, avec le formalisme, la réflexion théorique. Où cela se situe-t-il dans votre œuvre?

MiCHAEL DeLISLE Je pense qu'on écrit avec tout ce qu'on est, y compris ses lectures universitaires. Ce bagage, sans être nommé, passe dans les textes. On le voit tout de suite quand un écrivain a réfléchi à la langue, à la représentation, quand la voix est porteuse de conscience. Mais ces références (Derrida, Deleuze, Blanchot...) sont aussi des cautions morales et je ne suis pas un écrivain qui écrit avec son directeur de création au-dessus de l'épaule. Trop d'écrivains ont gâché leur talent avec cette inquiétude, cette servitude. J'ai beaucoup appris de Lise Tremblay là-dessus. Elle commence toujours ses séances d'écriture en disant: «Mangez tous d'la marde! » et elle a raison.

voix ET IMAGES Durant l'entretien, vous avez beaucoup évoqué vos parents et l'écriture d'une trame familiale. On a peu abordé la place du frère. Pourtant, la figure du frère est récurrente dans votre œuvre, tant dans les recueils de poésie que dans les romans.

MICHAEL DeLISLE Le frère de la poésie, ce n'est pas le frère du roman. Le frère de la poésie est une forme esthétisée de l'«autre». C'est le frère avec une connotation, disons, liturgique. Pour rester dans la sphère du précatif, c'est le prochain. Et c'est aussi le son «frère", c'est la musique de ce mot-là. Ce n'est donc pas nécessairement mon frère à moi que je vois dans ce mot en poésie. Mais dans le roman, oui, davantage. Mon frère réel contamine inévitablement le personnage du frère. Il fait partie des éléments que je cueille dans mon histoire familiale pour la transformer, la transmuer en mythe. On est dans la fabrication de paraboles transgénérationnelles. Ou plutôt d'une icône, d'un objet de culte qui sert à une méditation personnelle. Parce que l'histoire familiale a quelque chose d'imprenable, il faut la cerner. C'est ce que l'on disait à propos de la photo. Il faut tracer un cadre à la réalité pour que la révélation devienne possible. D'ailleurs, les artistes orthodoxes qui font les icônes ne parlent pas de peinture, ils disent qu'ils écrivent des icônes.

voIX ET IMAGES Cette métaphore de l'icône, avez-vous voulu l'intégrer à vos textes, en particulier à celui sur lequel vous travaillez actuellement?

Michael delisLe Non. Je ne veux pas en faire un thème. C'est plutôt un secret d'écriture. C'est lié à ma vie intime, à mon besoin de faire de mon histoire un objet qui soit de la nature d'une icône de bois. Je cherche à lui donner un format carré afin qu'elle ne soit plus un monstre, mais une image de monstre. Avec un petit lampion. 